\title{
Probabilistic Analysis and Design of a Raked Wing Tip for a Commercial Transport
}

\author{
Brian H. Mason*, Tzi-Kang Chen ${ }^{\dagger}$, Sharon L. Padula ${ }^{\star}$, Jonathan B. Ransom ${ }^{\S}$, and W. Jefferson Stroud ${ }^{* *}$ \\ NASA Langley Research Center, Hampton, VA, 23681-2199
}

\begin{abstract}
An approach for conducting reliability-based design and optimization (RBDO) of a Boeing 767 raked wing tip (RWT) is presented. The goal is to evaluate the benefits of RBDO for design of an aircraft substructure. A finite-element (FE) model that includes eight critical static load cases is used to evaluate the response of the wing tip. Thirteen design variables that describe the thickness of the composite skins and stiffeners are selected to minimize the weight of the wing tip. A strain-based margin of safety is used to evaluate the performance of the structure. The randomness in the load scale factor and in the strain limits is considered. Of the 13 variables, the wing-tip design was controlled primarily by the thickness of the thickest plies in the upper skins. The report includes an analysis of the optimization results and recommendations for future reliability-based studies.
\end{abstract}

\section{Introduction}

A. Motivation and Background

Optimization for structural sizing has been successful as a result of accurate, reliable, and computationally inexpensive finite-element (FE) methods for structural analysis (Refs. 1-5). However, the optimization process can generate designs that have poor off-design performance. Moreover, the optimization solution can be unduly influenced by design constraints that are not precisely known (Ref. 5).

A probabilistic approach is an attractive alternative to traditional design optimization. Probabilistic analysis and optimization can result in improved designs considering the variability of structural materials and the uncertainty in loads. Moreover, the process generates information as to which design changes have the biggest impact on reliability. This process gives the designer the added freedom, for example, to create lower cost designs with the same reliability as the baseline design or to produce higher reliability designs with a minimum addition of weight.

The current study explores the advantages of the probabilistic approach by choosing the Boeing 767-400ER raked wing tip (RWT) as a baseline design (see Figure 1). Unlike previous academic studies, this study starts with an FAA certified baseline design and suggests how it can be improved using probabilistic a design approach. To the extent possible, the study uses load cases and material characterizations that represent the as-built RWT. On the other hand, the design problem is necessarily simplified to expedite the study. The FE model is only appropriate for linear static analysis; therefore, crucial constraints like fatigue and damage tolerance are ignored. Similarly, only static-load cases are considered, and these load cases are not adjusted for changes in aerodynamic loading that result from changes in the design variables. The change in design variables is limited, so that changes in the aerodynamic loading can be neglected.

\section{B. Purpose and Contents}

The purpose of the RWT design study is to answer important questions about applying reliability-based design and optimization (RBDO) to aircraft design in general and, more specifically, to the design of the RWT. First, does a probabilistic approach produce a viable design? This question is answered by comparing RBDO designs with the baseline design. Next, does the probabilistic approach require exorbitant computer resources or measured data that are unavailable? This question is answered by estimating the computational costs of probabilistic analysis and establishing the needed and available data for the RWT.

In the limited context of structural analysis for a secondary substructure, this study shows the strengths and weaknesses of the RBDO approach. In this context, the benefit-to-cost improvement over the traditional design

\footnotetext{
* Research Aerospace Engineer, Durability, Damage Tolerance, and Reliability Branch, Senior Member AIAA

${ }^{\dagger}$ Senior Research Engineer, U. S. Army Research Lab, Vehicle Technology Directorate

*Senior Research Engineer, Aeronautics Systems Analysis Branch, Associate Fellow AIAA

${ }^{\S}$ Branch Head, Durability, Damage Tolerance, and Reliability Branch, Associate Fellow AIAA

${ }^{* *}$ Distinguished Research Associate, Durability, Damage Tolerance, and Reliability Branch, Associate Fellow AIAA
} 
approach is examined. Examples and recommendations are presented for characterizing and modeling uncertainties, for validating structural analyses and probability calculations, and for computing the final design risk while accounting for important uncertainties.

This paper is organized as follows. Section II provides a description of the RWT design problem. Section III presents details of the approaches that are used in the study. Section IV presents the results of the deterministic and probabilistic analyses for the baseline design. Section V presents the results of the deterministic and probabilistic design studies. A summary of the approaches that are evaluated and the conclusions of the design study are presented in Section VI. Finally, concluding remarks on the design study are presented in Section VII.

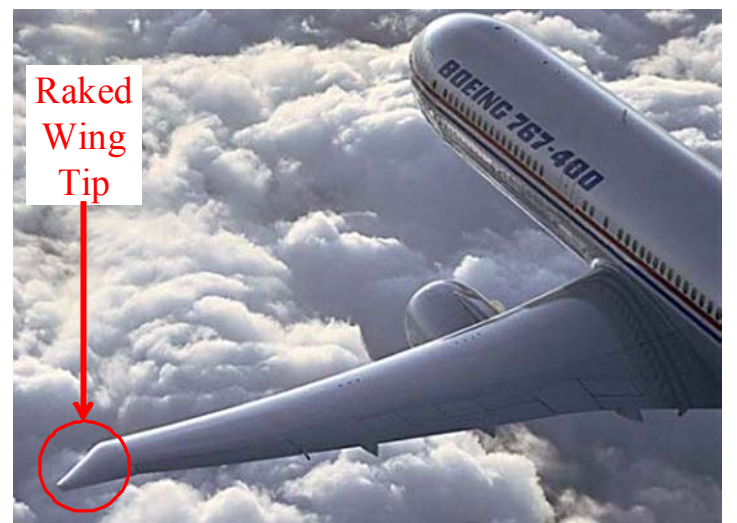

Figure 1. Boeing 767-400ER Raked Wing Tip.

\section{Description of RWT Design Problem}

In this section, the RWT design problem is described. The FE model and the analyses that simulate the RWT and its response are discussed. All FE analyses are performed with MSC/NASTRAN software, a product of MSC.Software Corporation (Ref. 6$)^{\dagger \dagger}$. Next, the design variables that are used to change the baseline design and the objective function that is used to assess the modified FE model are discussed. Finally, the deterministic and probabilistic design constraints are defined.

\section{A. Analysis model}

The FE model of the RWT consists of a metallic stubbed wing box and a composite RWT (Figure 2). Singlepoint displacement constraints are applied to the root of the stubbed wing box, so that the model acts like a cantilevered beam. The model consists of 2958 nodes, 3386 shell elements (CTRIA3, CQUAD4, and CSHEAR), 166 solid elements (CHEXA), and 501 line elements (CBAR, CBUSH, CELAS, and CROD).

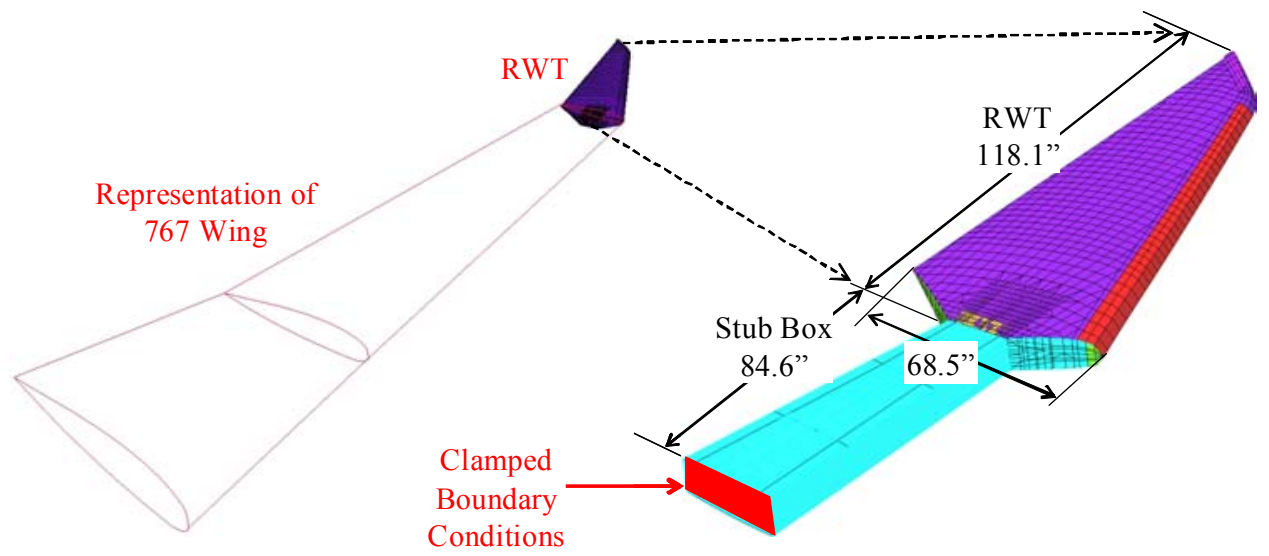

Figure 2. FE model of the stub box and the RWT.

\footnotetext{
${ }^{1 \dagger}$ The use of trademarks or names of manufacturers in this report is for accurate reporting and does not constitute an official endorsement, either expressed or implied, of such products or manufacturers by the National Aeronautics and Space Administration.
} 
As shown in Figure 2, the stubbed wing box is used to simulate constraints imposed by full wing box on the RWT. The focus of this study is the stiffness and strains in the RWT; so the structural response of the stub box is ignored in this study, and therefore, the elements of the stubbed wing box are not sized in the optimization process.

The RWT consists of four metallic components (i.e., root rib, root rib center section, leading edge, and tip rib, see Figure 3) and five composite components (i.e., upper cover, lower cover, forward spar, middle (half) spar, and aft spar, see Figure 4). The annotation (e.g. UC1, UC2, SM1, and SF1) in Figure 4 represents design variables used in the analysis and will be discussed later in the paper.

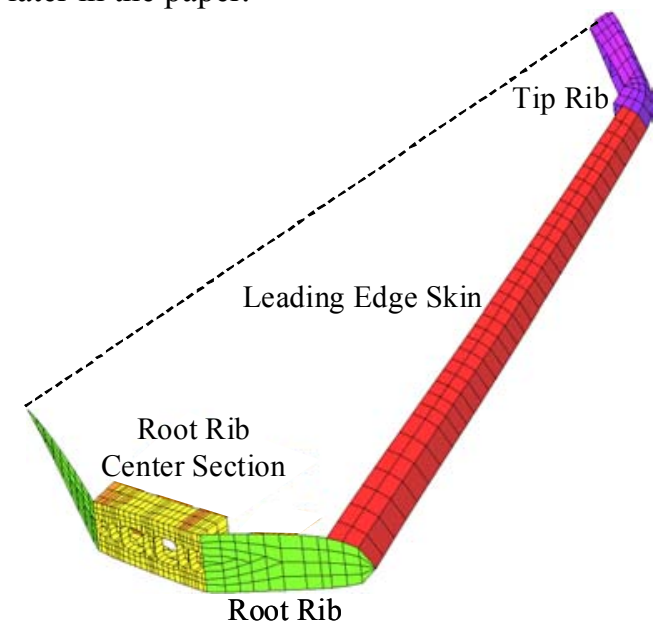

Figure 3. Metallic components in the FE model of the RWT.

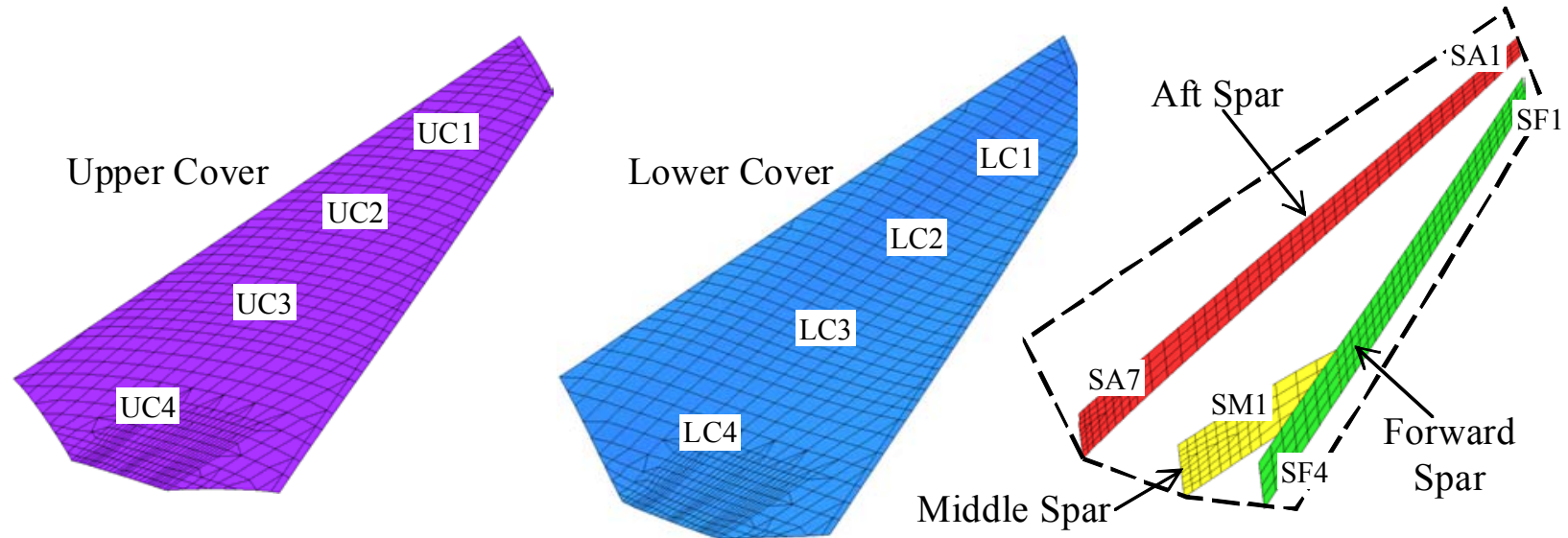

Figure 4. Composite components in the FE model of the RWT.

Structural engineers define limit loads as the largest load a structure is expected to encounter. Ultimate loads represent limit loads multiplied by a factor of safety. For this study, eight critical load cases are identified and applied to the FE model, as summarized in Table 1. The magnitude of the loads represents the ultimate loads for the deterministic design process.

Table 1. Load Cases

\begin{tabular}{|c|c|c|}
\hline Load case \# & Type & Altitude \\
\hline 1 & $1-\mathrm{g}$ spoilers down & Climb \\
\hline 2 & +2.5 -g spoilers up & Sea Level \\
\hline 3 & +2.0 -g flaps down & Sea Level \\
\hline 4 & +2.0 -g flaps down & Cruise \\
\hline 5 & $1.0-\mathrm{g}$ spoilers down & Sea Level \\
\hline 6 & Load case \#2 + buffet & Sea Level \\
\hline 7 & Load case \#4 + buffet & Sea Level \\
\hline 8 & Load case \#5 + buffet & Cruise \\
\hline
\end{tabular}


In the FE model, the composite shell properties are represented by NASTRAN PCOMP cards (Ref. 6). Each "ply" in the PCOMP card represents a layer of graphite epoxy fabric material. The material properties in each layer represent fabric as a smeared material system consisting of either a $0 / 90$ or a \pm 45 cross-ply. The exact properties of this material system are Boeing proprietary data, and hence are not presented in this report. The thickness of the covers is not constant; it varies in both the spanwise and chordwise directions. The exact thickness distribution is Boeing proprietary data, but in general the covers are thicker at the root of the RWT than at the tip. Similarly, the spars are thicker at the root than at the tip.

\section{B. Design Variables}

Thirteen design variables are defined for both deterministic and probabilistic optimization of the RWT. Each design variable is associated with the shell thicknesses of the composite components in the FE model as shown in Table 2. The design variables control the changes in thickness that are added to or subtracted from the original thickness for a given component (i.e., a value of 0.0 represents the baseline design). The lower limit of each design variable $\boldsymbol{\Delta} \boldsymbol{N}$ is equal to the minimum gage (MG) minus the baseline number of plies associated with that variable. Relative locations of the thirteen variables on the FE grid are shown in Figure 4. Multiple variables are used for each component as illustrated in Figure 5.

In Figure 5, variable UC1 represents a change in the thickness of the thinnest section of the upper cover, and variable UC4 represents a change in the thickness of the thickest section of the upper cover. The change in thickness at other sections of the upper cover is a piecewise linear function of the four design variables (UC1, UC2, UC3, and UC4) based upon the initial thickness of that section. During the optimization, lamina thicknesses in the PCOMP cards are changed as the design variables change. This approach is simpler to implement than adding layers and changing the stacking sequence. Although during the optimization process, the design variables are continuous; the design variables must be rounded up to the nearest integer number of plies for manufacturing purposes.

Table 2. Mapping of Design Variables to Composite Properties

\begin{tabular}{|c|c|c|c|c|}
\hline$\#$ & Label & Component & Baseline thickness (\# of plies $\boldsymbol{N}$ ) & Lower limit (\# of plies $\Delta \boldsymbol{N}$ ) \\
\hline 1 & UC1 & Upper cover & $M G+1$ & -1 \\
\hline 2 & UC2 & Upper cover & $M G+4$ & -4 \\
\hline 3 & UC3 & Upper cover & $M G+6$ & -6 \\
\hline 4 & UC4 & Upper cover & $M G+8$ & -8 \\
\hline 5 & LC1 & Lower cover & $M G+1$ & -1 \\
\hline 6 & LC2 & Lower cover & $M G+4$ & -4 \\
\hline 7 & LC3 & Lower cover & $M G+6$ & -6 \\
\hline 8 & LC4 & Lower cover & $M G+8$ & -8 \\
\hline 9 & SF1 & Forward spar & $M G+1$ & -1 \\
\hline 10 & SF4 & Forward spar & $M G+6$ & -4 \\
\hline 11 & SM1 & Middle spar & $M G$ & -6 \\
\hline 12 & SA1 & Aft spar & $M G+6$ & 0 \\
\hline 13 & SA7 & Aft spar & & -6 \\
\hline
\end{tabular}




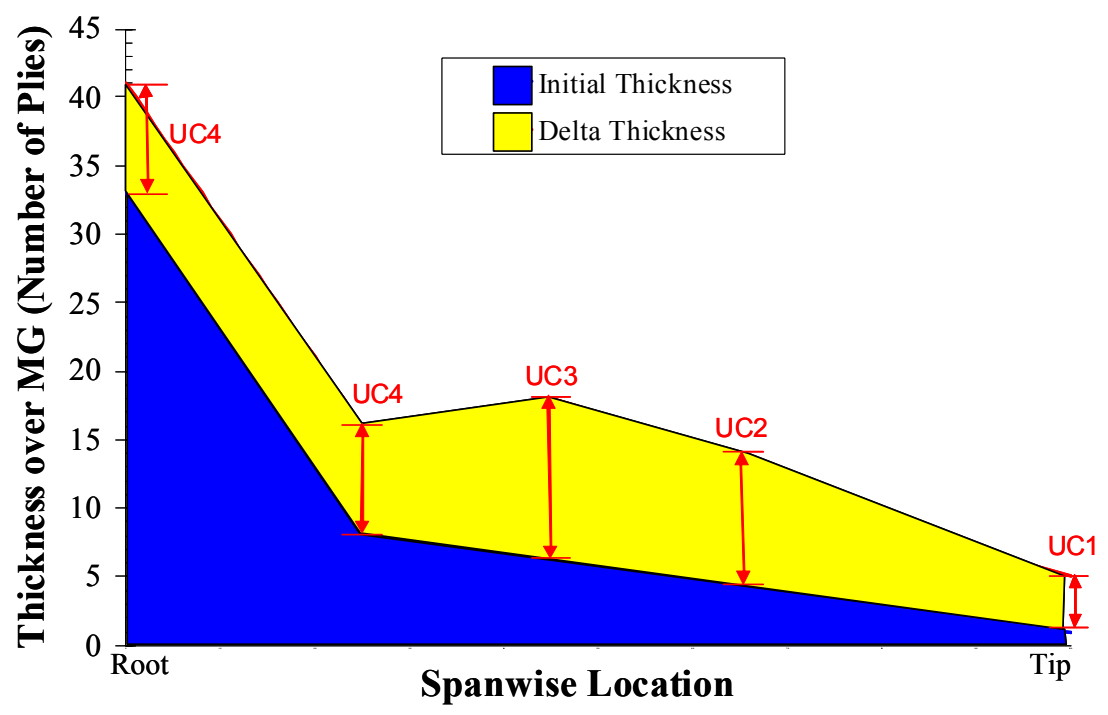

Figure 5. Sample mapping of design variables to ply thicknesses in upper cover.

For this study, only the lamina thickness is permitted to change. Several PCOMP cards in the upper and lower cover represent a sandwich composite and include a layer of core material. Preliminary parametric studies have determined that small changes in the thickness of the core material do not have a significant effect on the strains in the RWT model, so the thickness of the core layer in the upper and lower cover is not changed during the design process.

Special attention must be paid to beam elements on the spars of the FE model. In the forward, middle, and aft spars, shell elements represent the spar web, and beam elements represent the spar caps at the top and bottom of each spar as shown in Figure 6. For consistency in this study, the cross-sectional areas for the spar caps are linked to the thickness of the spar webs. In the actual RWT, the spars are C-channels; thus, the cross-sectional area $A$ for the spar caps (defined with NASTRAN PROD cards) in the FE model should be the product of the flange width $b$ and the spar web thickness. In the model parameterization process, constant flange widths for each PROD set are computed from the cross-sectional area and the initial thickness of the web connected to the beam elements associated with that PROD card. Therefore, the cross-sectional area for each PROD card is adjusted according to the value of the design variable for the spar web that is connected to the beam elements of that PROD card.

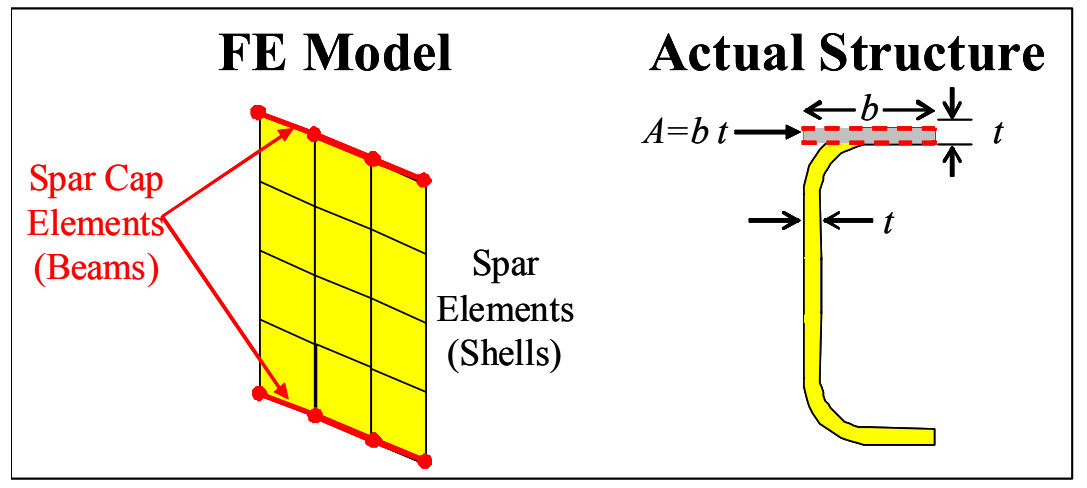

Figure 6. Cross-sectional dimensions of spar caps.

The conversion of the FE model into a parameterized FE model is an iterative process; thus, decisions such as whether to make the cross-sectional area of the spar cap a design variable or a computed quantity require some trial and error. In general, starting with design variables that map intuitively to the physical structure is advisable. In addition, variables should be normalized so that all have the same order of magnitude, and variables should be selected so that recovering the baseline case is easy. The set of design variables that is described here is one of many reasonable choices. 


\section{Objective function}

The objective of the optimization problem in this design study is to reduce the weight of the Boeing 767 RWT by changing the composite panel thicknesses and the spar cap cross-sectional areas (design variables). Weight is one output of the MSC/NASTRAN software. For this study, only the weight of the sized composite components is considered in the objective function. To eliminate the non-sized components from the weight calculation, the material densities of the non-sized components are set to zero.

\section{Constraints for deterministic optimization}

Three types of deterministic constraints are described in this section. The first constraint type is a side constraint that defines a lower limit on the design variables (i.e., $\Delta N$ in Table 2). The second constraint type is a modeling constraint that is based on the stiffness of the RWT. The third constraint type is a performance constraint that is based on composite strains and metallic stresses. This performance constraint has both a deterministic and a probabilistic formulation. The deterministic performance constraint is described in this section, and the probabilistic performance constraint is described in section II.F.

The purpose of the side constraints is to define a minimum gage for the composite material. Setting the minimum gage simplifies the margin of safety calculations and removes several failure modes related to impact resistance, durability, and damage tolerance.

The purpose of the modeling constraints is to limit the bending and twisting of the RWT so that the aerodynamic loads will not change due to excessive deformation of the RWT. These constraints are required in both the deterministic and probabilistic optimizations. In this study, bending stiffness and torsional stiffness of the RWT are defined by the displacements $w_{T i p}$ and $\alpha_{T i p}$ as shown in Figure 7. The constraints are feasible if the computed values of $w_{T i p}$ and $\alpha_{T i p}$ are within \pm 10 percent of the baseline values for each load case (i.e. the ratio of the computed value over the baseline value must be between 0.9 and 1.1 ). The $10 \%$ value was selected as a reasonable limit by the design team based on the experience of the loads engineers.

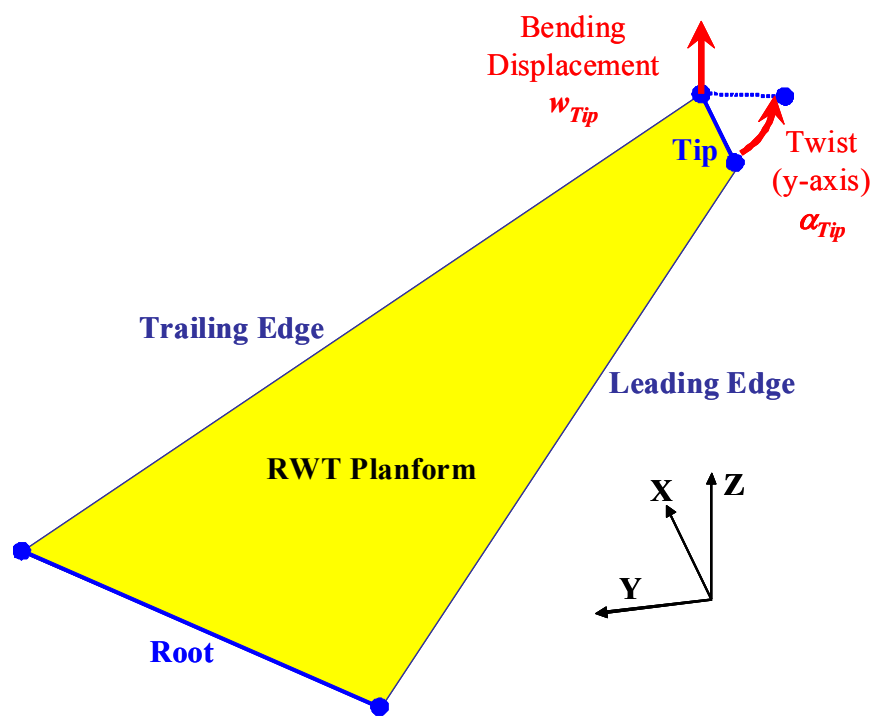

Figure 7. Stiffness constraints.

The purpose of the performance constraints is to assess whether the RWT is strong enough to resist the loads that are applied to it. The performance constraint for this design study is a margin of safety (MS) that is based on von Mises stresses and composite strains. The MS for the metallic components is computed for each element by:

$$
M S_{\text {Metallic }}=\frac{\sigma_{\text {vonMises }}^{\text {Allowable }}}{\sigma_{\text {vonMises }}^{\text {NASTRAN }}}-1
$$

where the computed value is the maximum of the upper and lower surface von Mises stress that is reported by MSC/NASTRAN, and the allowable value is the ultimate stress for the material that is associated with that element.

For composites, the MS should be computed for each of three failure modes: net section failure, bearing bypass interaction, and bearing failure. Unfortunately, the FE model does not include representative elements for bolts; 
thus, only the net section failure is considered in this study. The MS for composite components is computed for each ply in each element by:

$$
M S_{\text {Composite }}=\frac{1}{F I}-1
$$

where $F I$ is a composite failure index, which is computed with either a fiber tension equation (Eq. 3 ) or a fibermatrix shearing equation (Eq. 4), depending on the value of the axial strain $\varepsilon$.

$$
\begin{array}{ll}
F I=\frac{\varepsilon^{\text {NASTRAN }}}{\varepsilon^{T}} & \text { for } \varepsilon^{\text {NASTRAN }} \geq 0 \\
F I=\sqrt{\left(\frac{\varepsilon^{\text {NASTRAN }}}{\varepsilon^{C}}\right)^{2}+\left(\frac{\gamma^{\text {NASTRAN }}}{\gamma^{S}}\right)^{2}} & \text { for } \varepsilon^{\text {NASTRAN }} \leq 0
\end{array}
$$

In Eqs. 3 and 4, $\varepsilon^{\text {NASTRAN }}$ and $\gamma^{\text {NASTRAN }}$ represent NASTRAN-computed axial and shear strains, respectively. $\varepsilon^{T}, \varepsilon^{C}$, and $\gamma^{S}$ represent axial tension, axial compression, and shear strain limits, respectively. Axial strains are evaluated for both the 0/90 and the $+45 /-45$ fabric system, so there are two sets of axial strain limits. In Eqs. 3 and 4, the strain limits for the composite fabric material are proprietary Boeing data and hence are not presented in this paper

In the deterministic optimization process, only the lowest MS in each component for each load case is used. With 8 load cases and 9 components, 72 performance (i.e., MS) constraints are used in the design process. A summary of the deterministic constraints $(g)$ that are used in the deterministic design process is given in Table 3 .

Table 3. Summary of Deterministic Constraints

\begin{tabular}{|c|c|c|c|}
\hline Constraint type & Number of Constraints & Equation & Constraint \\
\hline Side (design variable) & 13 (1 per variable $)$ & $g_{1}(x)=x$ & $g_{1} \geq \Delta N$ \\
\hline Model (bending stiffness) & $8(1$ per load case $)$ & $g_{2}(x)=\frac{w_{\text {Tip }}^{\text {NASTRAN }}}{w_{\text {Tip }}^{\text {baseline }}}$ & $0.9 \leq g_{2} \leq 1.1$ \\
\hline Model (torsional stiffness) & $8(1$ per load case $)$ & $g_{3}(x)=\frac{\alpha_{\text {Tip }}^{\text {NASTRAN }}}{\alpha_{\text {Tip }}^{\text {baseline }}}$ & $0.9 \leq g_{3} \leq 1.1$ \\
\hline Performance (MS) & $\begin{array}{c}72(9 \text { per load case }) \\
\text { ultimate loads }\end{array}$ & $g_{4}(x)=M S_{\text {Ultimate }}$ & $g_{4} \geq 0$ \\
\hline
\end{tabular}

\section{E. Probabilistic Variables}

One of the key issues in probabilistic design is to determine the uncertainties that are associated with structural analysis. The type, distribution, sensitivity, and significance for each source of uncertainty must be considered. Possible sources of uncertainty include modeling errors, manufacturing errors, environmental variability, aerodynamic loads, and material variability. Many sources of uncertainty were ignored for this study because their effects on the design were considered small in comparison to other sources of uncertainty or because of a lack of quantifiable data on the uncertainty distributions. The ignored sources of uncertainty include material elastic properties, as-manufactured cover and spar thicknesses, model fidelity errors, and analytic simulation errors.

Based on prior research experiences in structural analysis, two major sources of uncertainty dominate the failure probability (Refs. 2-4). These two uncertainties are randomness in the applied load and variability in the allowables (i.e., strength and strain limits). The procedure that is used to estimate the distributions for these two sources of uncertainty is discussed in the following section.

\section{E.1 Distribution for the load}

Load distribution is difficult to determine in the absence of measured data. For aerospace vehicles, the loads depend upon many factors (e.g., flight path, accelerations, payload, gusts, wind, aeroelasticity, maneuvering, airspeed, and vibration). The uncertainty of the load is the combination of the randomness in all of these factors. In the traditional design method, the loads are determined based on the airplane configuration and the aerodynamic performance as specified by the design flight envelopes. This study lacked the resources required for the in-depth study of the load spectrum which would be required to develop a refined load uncertainty distribution. However, the data and calculations that are presented in Ref. 7 provide evidence that such a distribution can be developed. 
A practical method was applied to estimate the load and its uncertainty based on the knowledge of the RWT study team members. The FE model that was provided by Boeing includes eight load cases that represent ultimate loads for eight critical flight conditions (see Table 1). These loads can be scaled to design limit loads (DLL's) by dividing the NASTRAN load scale factor by 1.5. Aircraft designers assume that DLL's are the maximum loads that are experienced by the Boeing 767-400ER aircraft during its service life of 40,000 flights. Designers assume that the occurrence of 100-percent DLL is relatively rare. A conservative estimate is that DLL occurs once in 3,000 flights. However, the occurrence of 75-percent DLL is not rare, perhaps once in two flights. These assumptions are captured in a probabilistic distribution that is shown in Figure 8. Here, a normal distribution is used to model the uncertainty, and the distribution is normalized so that the DLL has a value of unity. Notice that Figure 8 represents the probabilistic distribution of the maximum load per flight rather than the distribution of the load. Any probability of failure calculated using this distribution is the probability of failure per flight.

In this study, a normal distribution is selected for the loads because the central limit theorem states that the combination of the uncertainties of multiple factors is a normal distribution (Ref. 8). For a standard normal distribution (i.e., mean $=0$, standard deviation $=1$ ), the notation $\Phi(\mathrm{z})(\mathrm{Eq} .5)$ is commonly used to represent the distribution function of the standard normal variate $K$. The standard normal variate represents the number of standard deviations from the mean $\mu$ (Ref. 8). The value for the standard normal variate $K_{p}$ for a cumulative probability $p$ is computed from Eq. 6:

$$
\begin{aligned}
& \Phi(z)=\frac{1}{\sqrt{2 \pi}} \int_{\xi=-\infty}^{z} e^{\left(-\frac{1}{2} \xi^{2}\right)} d \xi \\
& K_{p}=\Phi^{-1}(p)
\end{aligned}
$$

If the cumulative probability that the maximum load per flight exceeds the DLL is assumed to be $1 / 3000$ or $3.333 \times 10^{-4}$, then the standard normal variate $K_{p}$ is calculated to be 3.402933 . The value $L$ (percent of DLL) for a given $K_{p}, \mu$, and coefficient of variation (COV) can be computed as:

$$
L=\mu \cdot\left(1+C O V \cdot K_{p}\right)
$$

Thus, if we assume a COV of 10 percent, then the mean for the maximum load per flight (100\% DLL) can be computed by using Eq. 7 to be 0.7461 and is pictured in Figure 8 . Notice that the mean value of the load scale factor is about 75 percent of the DLL, which is consistent with the assumptions that were provided by the aircraft designers involved in this study.

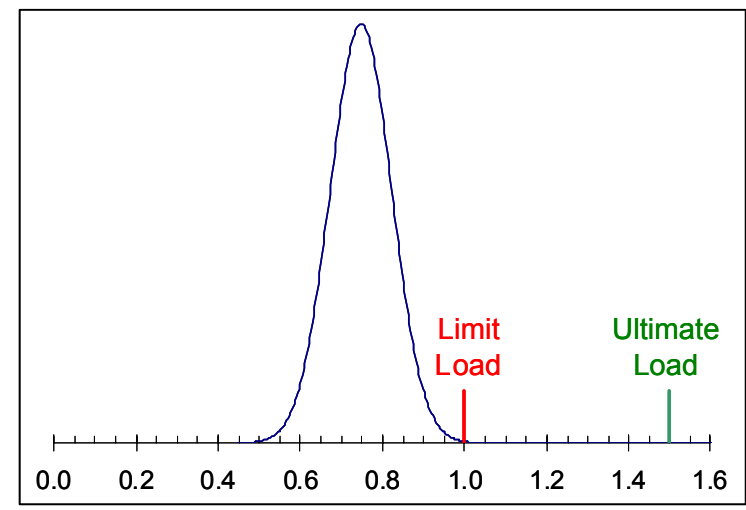

Figure 8. Normal distribution for load scale factor of the maximum load per flight.

\section{E.2 Distribution for material strength and strain limits}

Strength and strain limits of a material system are commonly described using three types of values: A-basis, Bbasis and typical $(\mu)$ values. The use of A-basis and B-basis allowable properties recognizes that material properties are statistical in nature. For this study, B-basis tolerance bounds are used to characterize uncertainties in the material system. The B-basis tolerance is the value above which 90 percent of a specified population of measurements is expected to fall, with a confidence of 95 percent (Refs. 9 and 10).

To obtain the material variation, multiple test samples from multiple batches and panels are collected to include variations from sample to sample, panel to panel, and batch to batch. The B-basis $\left(R_{B}\right)$ of the materials can be 
calculated using the mean $\mu_{s}$ and the coefficient of variation $C O V_{s}$ (standard deviation $S D_{s}=C O V_{s}^{*} \mu_{s}$ ) of the testing sample as:

$$
R_{B}=\mu_{s} \cdot\left(1-K_{B} \cdot C O V_{s}\right)=\mu_{s}-K_{B} \cdot S D_{s}
$$

where $K_{B}$ is a tolerance limit factor. The tolerance limit factors are functions of the distribution and sample size as shown in Table 4 (data from Ref. 10, chapter 8). Note that the values for the infinite sample size are obtained from Equation 6, as shown in Figure 9.

Table 4. Tolerance Limit Factors for Normal Distribution

\begin{tabular}{|c|c|}
\hline Sample size & $\boldsymbol{K}_{\boldsymbol{B}}$ \\
\hline 5 & 3.408 \\
\hline 10 & 2.355 \\
\hline 30 & 1.778 \\
\hline 100 & 1.527 \\
\hline infinite & 1.282 \\
\hline
\end{tabular}

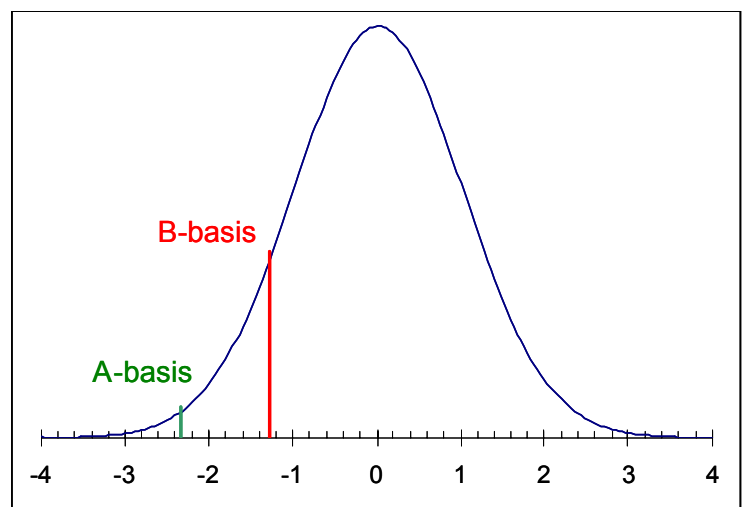

Figure 9. Normal distribution for material limits assuming infinite sample size.

For the RWT study, a normal distribution is assumed for the strain limit distributions. The mean value $\mu$ and the B-basis $\left(R_{B}\right)$ of the material were provided by Boeing, but the coefficient of variation $C O V$ of the material was not available. If the sample size is given, the $C O V$ and $S D$ can be computed from Equation 9. The number of samples used to determine the $R_{B}$ ranges from 5 to 30 specimens. Since the exact number of samples used to determine the $R_{B}$ ranges is not available in this study, an additional analysis using Chi-Square distribution (Ref. 10) and sample size was conducted for $95 \%$ high confidence bound of the $S D$ of samples. Because the $95 \%$ high confidence bound of the $S D$ computed for 5 and 30 samples differ by less than four percent, the SD calculated with sample size 5 is used for this study. The resulting $C O V$ values for the composite fabric are presented in Table 5.

Table 5. Probabilistic Distributions for Composite Strain Limits

\begin{tabular}{|c|c|}
\hline Strain type & COV \\
\hline $0^{\circ} / 90^{\circ}$ fabric tension & 0.155 \\
\hline $0^{\circ} / 90^{\circ}$ fabric compression & 0.118 \\
\hline$+45^{\circ} / 45^{\circ}$ fabric tension & 0.155 \\
\hline$+45^{\circ} / 45^{\circ}$ fabric compression & 0.118 \\
\hline Shear & 0.100 \\
\hline
\end{tabular}

\section{F. Constraints for probabilistic optimization}

Three types of constraints are used in the RBDO process. The first two constraint types, side constraints and model constraints, are deterministic and were discussed in section II.D. The third constraint type is a probabilistic performance constraint, which is described in this section.

A summary of all of the constraints used in the RBDO process is given in Table 6. The probabilistic performance constraint $\left(g_{4}\right)$ is based on the idea that a MS that is less than zero is unacceptable. In this study, the probability of failure $P_{f}$ means the probability that $\mathrm{MS}_{\text {Limit }}$ is less than zero (i.e., $\mathrm{P}\left[\mathrm{MS}_{\text {Limit }}<0\right]$ ). Here, the $\mathrm{MS}_{\text {Limit }}$ 
is computed as the minimum MS for all components for all load cases (i.e the minimum of $72 \mathrm{MS}$ responses). In Table 6, the constraint $g_{4}$, is that the $P_{f}$ must be smaller than a prescribed system probability $P_{R e q}$. In this study, Monte Carlo simulation is used to compute $P_{f}$.

Table 6. Summary of Probabilistic Constraints

\begin{tabular}{|l|c|c|}
\hline \multicolumn{1}{|c|}{ Constraint type } & Number of Constraints & Constraint \\
\hline Side (design variable) & $13(1$ per variable $)$ & $g_{1} \geq \Delta N$ \\
\hline Model (bending stiffness) & $8(1$ per load case $)$ & $0.9 \leq g_{2} \leq 1.1$ \\
\hline Model (torsional stiffness) & $8(1$ per load case) & $0.9 \leq g_{3} \leq 1.1$ \\
\hline Performance (MS) & 1 & $g_{4}=P_{f}=P\left(M S_{\text {Limit }}<0\right) \leq P_{r e q}$ \\
\hline
\end{tabular}

\section{Design Approaches}

Each design approach combines a FE analysis tool (MSC/NASTRAN, Ref. 6) and an optimizer (Design Optimization Tools (DOT), Ref. 11) with additional NASA-developed codes to perform the data transfer, the response surface approximations, and the probabilistic analysis. This set of codes was integrated using commercial framework software called ModelCenter ${ }^{\mathbb{R}}$, a product of Phoenix Integration, Inc. (Ref. 12). In the following subsections, the approaches for the deterministic design and the probabilistic design are described.

\section{A. Deterministic design approach}

In the deterministic optimization, the 13 design variables that are listed in Table 2 are adjusted to reduce the weight of the composite components subject to the 13 side constraints, 16 model constraints, and 72 performance constraints that are listed in Table 3. A functional flow diagram of the deterministic optimization is illustrated in Figure 10. As shown in the flowchart, the "Compute Responses" phase uses MSC/NASTRAN to compute the objective function and constraints, and the "Optimization Search" phase uses the optimizer DOT to adjust the values of the design variables. The process continues to iterate between compute and optimization phases as long as the design variable, constraint and objective values are changing. The process can also terminate if the number of analyses exceeds some maximum number (e.g., 300 is the maximum number allowed for the RWT study). Each structural analysis (using load cases 1 through 8) requires 21 seconds of CPU time on a single node of a $2.0 \mathrm{GHz}$ Pentium IV Linux cluster. A typical optimization requires less than 300 analyses and is completed in a few hours. Standard approximate analysis techniques and parallel processing strategies can be used to reduce the computation time but were deemed unnecessary for this study.

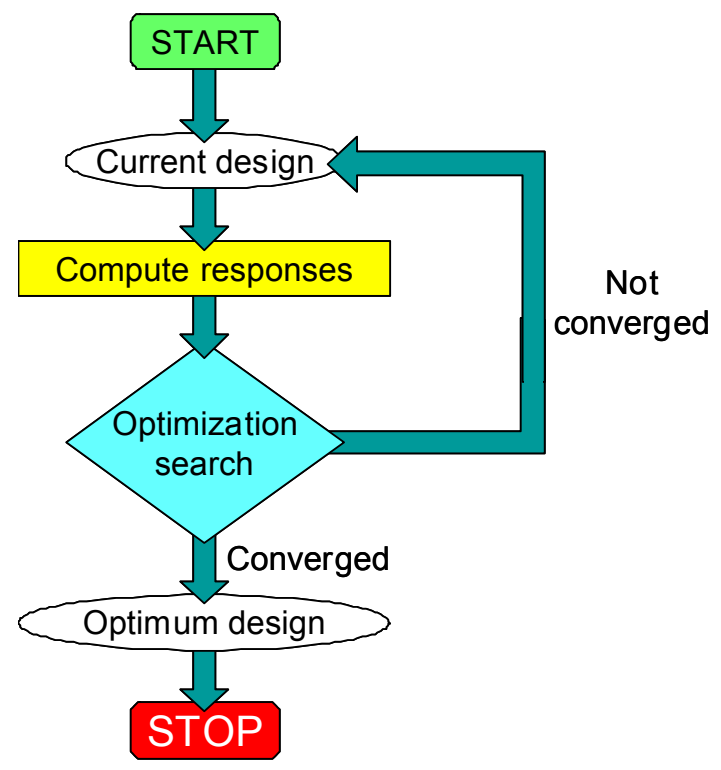

Figure 10. Functional flow diagram for deterministic optimization. 


\section{B. Probabilistic design approach}

The probabilistic design problem is defined in much the same way as the deterministic design problem. The 13 design variables that are listed in Table 2 are adjusted to reduce the weight of the composite components subject to the side, model, and performance constraints that are listed in Table 6 . The difference between the two design approaches is that the 72 deterministic performance constraints are replaced by a single probabilistic constraint.

In the probabilistic approach, the structural analysis is performed with inputs that are uncertain in nature. Random number values for the strain limits and the load scale factors are generated using an algorithm that is described in Ref. 13. Because the NASTRAN load cards are defined as ultimate loads in the deterministic optimization, the random load scale factor that is used in the probabilistic calculation is reduced by a factor of 1.5 to represent limit loads.

The probability $P_{f}$ that $\mathrm{MS}_{\text {Limit }}<0$ is estimated by Monte Carlo (MC) simulations. This choice is justified because 72 MS functions define the failure region, and efficient methods, such as First Order Reliability Method (FORM), are known to underestimate the value of $P_{f}$ when a large number of limit state functions exist (Ref. 5). However, the MC method is computationally expensive; thus, the number of NATRAN calculations must be limited, and the use of approximate analysis must be considered.

The number of MC simulations needs to be as large as possible. A large number of MC simulations is more likely to accurately reflect the distribution of the underlying random variables and is more likely to provide an accurate prediction of $P_{f}$. In addition, the DOT optimizer requires derivatives of $P_{f}$ with respect to the design variables. In $\mathrm{MC}$ simulation, $P_{f}$ is computed from an integer count of the number of simulations that fail divided by the total number of simulations performed, $N_{S}$. Therefore, the resolution of the $P_{f}$ calculation is $1 / N_{S}$. This resolution must be sensitive enough such that both the values and derivatives of $P_{f}$ are accurate.

The error that is associated with a MC simulation can be estimated using a "confidence interval." A confidence interval is defined as a range of values for $P_{f}$ that is likely to contain the true value of $P_{f}$ (Ref. 9). For a normal distribution, the 95-percent confidence interval (CI) is defined as:

$$
C I=1.96 \sqrt{\frac{1-P_{f}}{P_{f} \cdot\left(N_{S}-1\right)}}
$$

where $P_{f}$ is computed by the MC simulation. For the small values of $P_{f}$ that are computed in the present study, Eq. 10 can be rewritten as

$$
P_{f} \cdot N_{S} \approx\left(\frac{1.96}{C I}\right)^{2}
$$

Then, a value of $P_{f} \cdot N_{s}=500$ guarantees a CI of approximately 9 percent. For this study, all reported results have a CI of $9 \%$. Thus, $10^{8}$ sampling points are needed when the $P_{\text {req }}$ is $5.0 \mathrm{e}-6$.

The use of response surface methods or parallel processing is not needed to speed up the MC simulation because the design variables are not random variables and the NASTRAN analysis is linear static. Therefore, only one structural analysis is required to estimate all of the values that are needed in the MC simulation. An MC simulation with one hundred million $\left(10^{8}\right)$ sampling points using all 28 strain limits and all 8 random loads requires 25 minutes on a single node of a $2.0 \mathrm{GHz}$ Pentium IV Linux cluster. For this study, DOT required an average of 160 function evaluations for convergence. The computation time for each converged optimization process ranged from less than a minute to several days depending on the value of $P_{\text {req. }}$.

To validate the $\mathrm{MC}$ simulation results, additional probabilistic analysis methods were performed for the present study. First, a probabilistic design study is performed with only one random variable. For this case, the system $P_{f}$ was computed by:

$$
P_{f}=P\left(\mathrm{MS}_{\text {Limit }}<0\right)=\frac{1}{C O V \cdot \mu \sqrt{2 \pi}} \int_{z=-\infty}^{z_{0}} e^{-\frac{1}{2}\left(\frac{z-\mu}{\operatorname{COV} \cdot \mu}\right)^{2}} d z
$$

where $P_{f}$ is computed from the nominal limit load MS, COV is the coefficient of variation, $\mu$ is the mean of the random value, and $z_{0}$ is the nominal value of the random value.

\section{Analysis Results for Baseline Design}

In this section, the results of the deterministic and probabilistic analyses of the baseline design of the RWT are presented. In the baseline design, the design variables shown in Table 2 have values of zero. Deterministic results computed include weight and margin of safety (MS). Probabilistic results include $P_{f}$ computed using both MC 
simulation and exact analysis with several combinations of the 8 random load scale factors and 28 random strain limits.

\section{A. Deterministic Analysis}

The weight of the FE model is computed by NASTRAN using the component material densities and the element dimensions. A weight breakdown of the FE model by component is presented in Table 7. The weight of the stubbed wing box is included in Table 7 because this component is only a simplistic representation of the actual 767 wing box and only serves as a region for the application of the displacement constraints so that the RWT acts like a cantilever beam. Because the geometry (i.e., the FE node locations) of the RWT is fixed, the weight of the RWT is a perfectly linear function of the design variables. The weight serves as the objective for both the deterministic and probabilistic design studies.

Table 7. Baseline Component Weight Breakdown

\begin{tabular}{|l|c|}
\hline \multicolumn{1}{|c|}{ Component } & Normalized Weight \\
\hline Composite skin, upper & 0.402 \\
\hline Composite skin, lower & 0.397 \\
\hline Front spar webs & 0.044 \\
\hline Front spar caps (chords) & 0.042 \\
\hline Middle spar webs & 0.025 \\
\hline Middle spar caps (chords) & 0.016 \\
\hline Aft spar webs & 0.041 \\
\hline Aft spar caps (chords) & 0.033 \\
\hline Total & $\mathbf{1 . 0 0 0}$ \\
\hline
\end{tabular}

The deterministic performance constraints are computed from the margins of safety for both metallic and composite components. MS results for the composite components are presented in Figures 11 and 12. For composite components, the B-basis strain limits are used to compute the MS for the deterministic constraints. In Figure 11, the most critical (i.e., the lowest) MS in each composite component is plotted for each load case for the baseline design. For load case \#1, the lowest MS occurs in the middle spar. For load cases 2 through 8, the lowest MS occurs in the upper cover. From Figure 11, the most critical load case is \#6 with a MS of 0.126. A fringe plot of the MS in the composite components is presented in Figure 12.

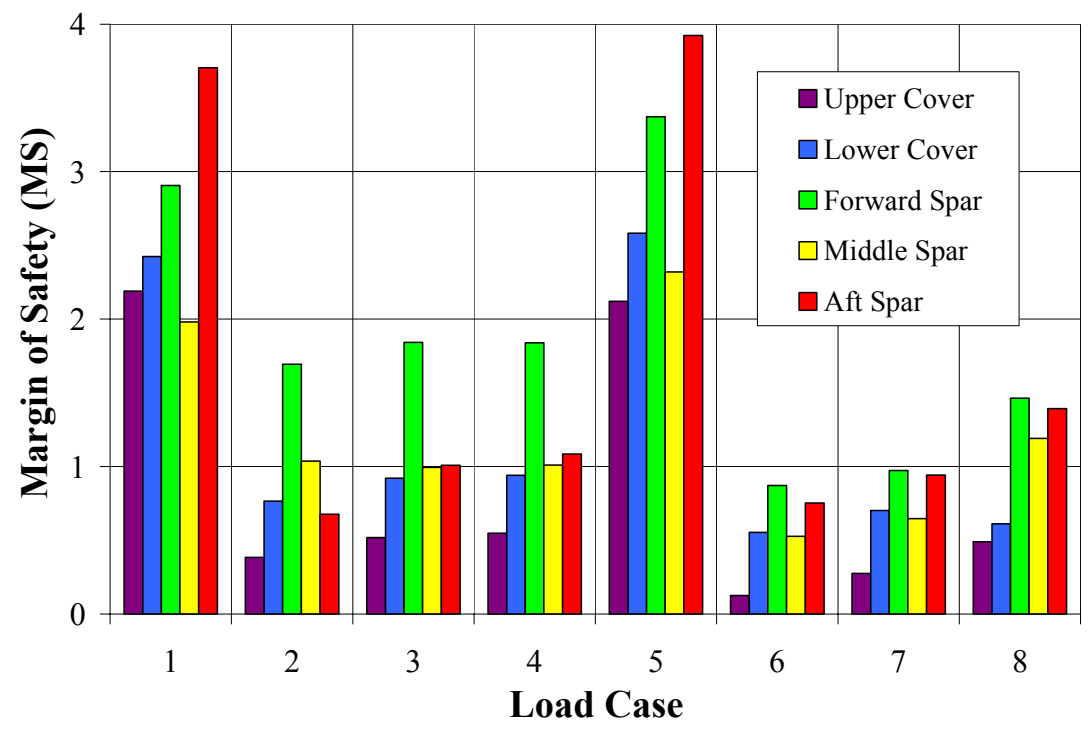

Figure 11. MS in composite components under ultimate load. 

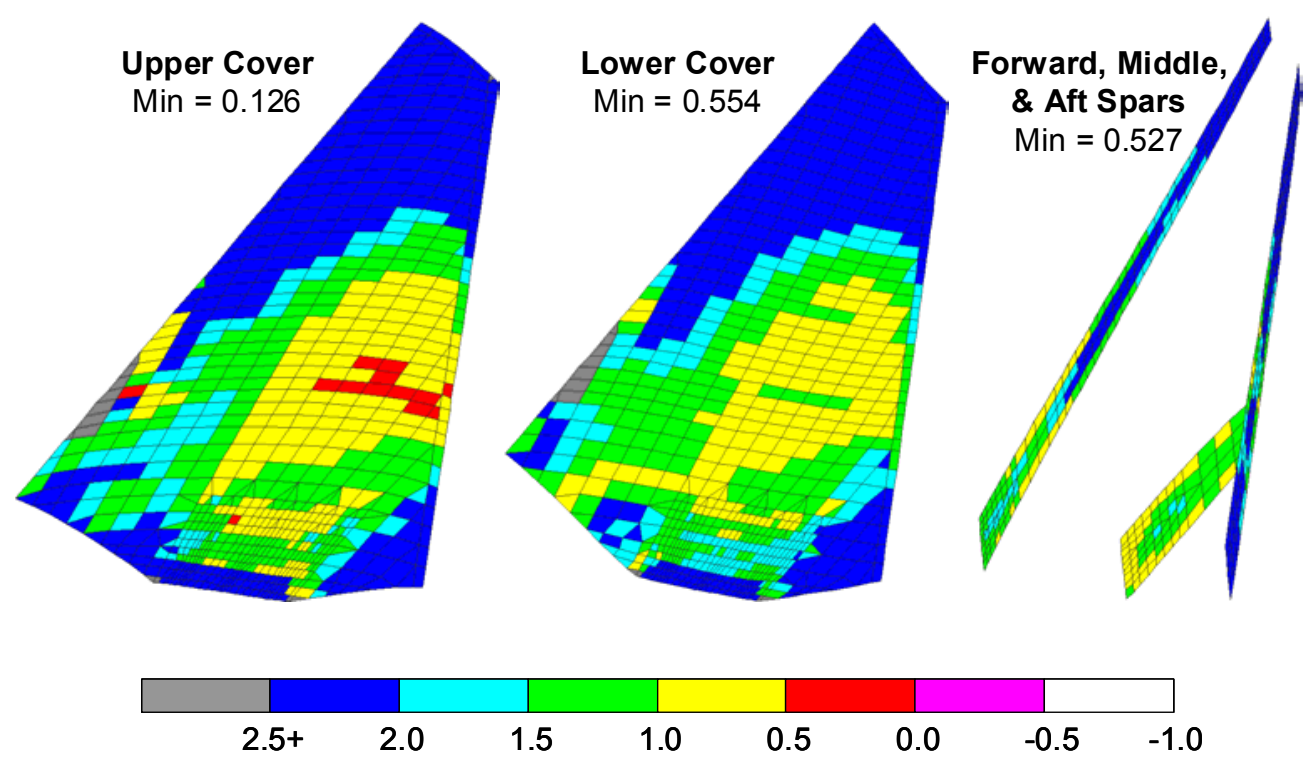

Figure 12. MS in composite components for most critical load case (\#6).

\section{B. Probabilistic analysis}

The only probabilistic constraint in the design process is a MS-based performance constraint $P_{f}=P\left(\mathrm{MS}_{\text {limit }}<0\right)$. In this section, the effects of different random variables on $P_{f}$ are evaluated. First, the randomness in the material limits is considered; then, randomness in the load scale factor is considered. Finally, the effect of applying both the random material limits and the load scale factors is evaluated. In the single random variable cases, the MC simulation results are verified against the results of other methods. In the following tables, results for MC simulations with three different sample sizes are compared to determine the sample size necessary for accuracy.

Three cases are considered using only random distributions on material limits, as given in Table 8 . In these cases, the fixed load scale factor that corresponds to the DLL is used. With only one random value, $P_{f}$ can be computed using Equation 12. If only randomness in the strain limit of the upper cover (the component with the lowest MS) is considered, then the $P_{f}$ from Eq. 12 is $6.343 \mathrm{e}-6$, which is 4.36 standard deviations away from the mean. As shown in Table 8, the use of multiple strain limits causes a significant increase in $P_{f}$.

Table 8. Calculation of $\boldsymbol{P}_{f}$ with only Random Material Limits

\begin{tabular}{|l|c|c|c|c|}
\hline \multicolumn{1}{|c|}{ Random variables } & \multirow{2}{*}{ Eq. 12 } & \multicolumn{3}{c|}{ MC sample size } \\
\cline { 3 - 5 } & & $\mathbf{1 0}^{\mathbf{7}}$ & $\mathbf{1 0}^{\mathbf{8}}$ & $\mathbf{5 \cdot 1 0}^{\mathbf{8}}$ \\
\hline One strain limit (0/90 compression) & $6.343 \mathrm{e}-6$ & $5.9 \mathrm{e}-6$ & $5.93 \mathrm{e}-6$ & $5.846 \mathrm{e}-6$ \\
\hline Two strain limits (0/90 compression and shear) & N/A & $6.4 \mathrm{e}-6$ & $5.74 \mathrm{e}-6$ & $5.742 \mathrm{e}-6$ \\
\hline All 28 strain limits & N/A & $3.53 \mathrm{e}-5$ & $3.380 \mathrm{e}-5$ & $3.377 \mathrm{e}-5$ \\
\hline
\end{tabular}

Two cases are considered with only random distributions on the load scale factor, as given in Table 9. In Table 9, the limit load distribution for the first case corresponds to a probability of exceeding the DLL of 3.3e-4 per flight. For the first case, the $P_{f}$ from Eq. 12 is $6.59 \mathrm{e}-37$, which is 12.6 standard deviations away from the mean. A second case that represents a 50-percent probability of exceeding the DLL is presented in Table 9 to illustrate the effects of a different load distribution on the $P_{f}$ response. Even with the second extreme load distribution, the probability of failure is quite small. The $P_{f}$ that is attributable only to randomness in the load scale factor is low, but the inclusion of the effects of random load scale factors in the RBDO process is important.

Table 9. Calculations of $\boldsymbol{P}_{f}$ with only a Random Load Scale Factor

\begin{tabular}{|c|c|c|}
\hline Limit load distribution (load case \#6) & Eq. 12 & MC (sample size $=\mathbf{5} \cdot \mathbf{1 0}^{\mathbf{8}}$ ) \\
\hline Normal, $\mu=0.7461$ & $6.59 \mathrm{e}-37$ & 0 \\
\hline Normal, $\mu=1.0000$ & $2.80 \mathrm{e}-12$ & $2.79 \mathrm{e}-12$ \\
\hline
\end{tabular}

Three cases are considered with random distributions in both strain limits and load scale factors, as given in Table 10. In the first and second cases, only strains for load case $\# 6$ are considered. In the last two cases, 
uncertainties in strain limits for all load cases are considered, but different load distributions are used. The addition of more random distributions in the design process results in an increase in $P_{f}$ from 2.e-8 to 1.2e-6. Comparison of the results in Tables 8 and 10 reveals that the addition of one random load reduces $P_{f}$ by a factor of 30 over the random strains alone.

Table 10. Calculations of $\boldsymbol{P}_{f}$ with Both Random Material Limits and Load Scale Factors

\begin{tabular}{|l|c|c|c|c|}
\hline \multicolumn{1}{|c|}{ Strain limit distributions } & Load distributions & \multicolumn{3}{|c|}{ MC sample size } \\
\cline { 3 - 5 } & & $\mathbf{1 0}^{\mathbf{7}}$ & $\mathbf{1 0}^{\mathbf{8}}$ & $\mathbf{5 \cdot 1 0}^{\mathbf{8}}$ \\
\hline One strain limit (0/90 Compression) & Load case \#6 (Normal) & 0 & $2 . \mathrm{e}-8$ & $1.6 \mathrm{e}-8$ \\
\hline All 28 strain limits & Load case \#6 (Normal) & $6 . \mathrm{e}-7$ & $5.2 \mathrm{e}-7$ & $5.76 \mathrm{e}-7$ \\
\hline All 28 strain limits & All load cases (Normal) & $9 . \mathrm{e}-7$ & $1.21 \mathrm{e}-6$ & $1.174 \mathrm{e}-6$ \\
\hline
\end{tabular}

\section{Results of the design studies}

In this section, the results of the deterministic and the probabilistic optimization studies are presented. In the deterministic optimization study, three optimization runs are conducted with different initial designs. Also, the results for a deterministic optimization with discrete design variables are presented. In the RBDO study, several optimization runs are performed with a different target $P_{\text {req }}$ to study the cost (in terms of weight) of increasing the required reliability of the RWT. Finally, the sensitivities of the responses to the design variables are compared to evaluate the robustness of the deterministic design.

\section{A. Deterministic optimization}

For the initial deterministic optimization, three optimization runs are conducted with three starting designs: the baseline Boeing design, a minimum weight design, and a maximum weight design. The optimization is performed with a version of the DOT software that is embedded within the ModelCenter framework. The optimum weights for these three starting points are close (within $0.2 \%$ ). Actual fabrication of the RWT requires an integer number of plies; thus, the nearest optimum design with discrete (integer) design variables is determined by a parametric study. The resulting optimum design is presented in Tables 11 and 12. Notice from the yellow shading in Table 11 that only five variables (three in the upper cover, one in the lower cover, and one in the aft spar) have values that are greater than the lower bounds. The optimum design was $83.48 \%$ of the weight of the baseline design. For the optimum design, the twist ratio constraint was active (near the $110 \%$ stiffness limit) for load case \#8, and the margin of safety constraint ( $\mathrm{g}_{4}$ from Table 3 ) was nearly active (almost zero) in the upper cover for load case \#6.

Table 11. Deterministic Optimum Designs for Discrete Design Variables

\begin{tabular}{|c|c|c|c|c|}
\hline$\#$ & Label & Lower limit, $\boldsymbol{\Delta N}$ & Design variable values & Number of plies \\
\hline 1 & UC1 & -1.0 & -1.0 & MG - 1.0 \\
\hline 2 & UC2 & -4.0 & -4.0 & MG - 4.0 \\
\hline 3 & UC3 & -6.0 & -3.0 & MG - 3.0 \\
\hline 4 & UC4 & -8.0 & -2.0 & MG - 2.0 \\
\hline 5 & LC1 & -1.0 & -1.0 & MG - 1.0 \\
\hline 6 & LC2 & -4.0 & -4.0 & MG - 4.0 \\
\hline 7 & LC3 & -6.0 & -3.0 & MG - 3.0 \\
\hline 8 & LC4 & -8.0 & -8.0 & MG - 8.0 \\
\hline 9 & SF1 & -1.0 & -1.0 & MG - 1.0 \\
\hline 10 & SF4 & -4.0 & -4.0 & MG - 4.0 \\
\hline 11 & SM1 & -6.0 & -6.0 & MG - 6.0 \\
\hline 12 & SA1 & 0.0 & 0.0 & MG - 1.0 \\
\hline 13 & SA7 & -7.0 & -1.0 & \\
\hline
\end{tabular}

Table 12. Objective and Constraint Values for Discrete Design Variables

\begin{tabular}{|l|c|c|}
\hline \multicolumn{1}{|c|}{ Response } & Baseline & Discrete optimum \\
\hline Normalized Weight & 1.0000 & 0.8348 \\
\hline Twist ratio for load case \#8 & 1.0000 & 1.0999 \\
\hline MS (UC for load case \#6) & 0.1260 & 0.00563 \\
\hline
\end{tabular}




\section{B. Probabilistic optimization results}

For the RBDO study, several optimization runs are conducted using different values of the $P_{\text {req }}$ (from $1.0 \mathrm{e}-3$ to 5.0e-7) as constraints. Again, the optimizations are performed using the DOT software within ModelCenter framework. In Figure 13, the optimized weights for the different target values of $P_{\text {req }}$ are plotted. Figure 13 illustrates the cost of requiring a more stringent probability of failure. For example, if the $P_{\text {req }}$ constraint for the RWT is decreased from $10^{-6}$ to $10^{-5}$, then the optimum weight of the RWT could be reduced by 10 pounds. The relationship between the optimized weight and the $\log$ of the $P_{r e q}$ is approximately linear for values of $P_{\text {req }}$ between 5.e-7 and 5.e-5. When $P_{r e q}$ is greater than 1.e-4, the deterministic stiffness constraint begins to drive the design. A deterministic optimization that is performed with only the stiffness constraint is also shown in Figure 13. For this deterministic design, the corresponding probability of failure is computed as $1.07 \mathrm{e}-3$. Because the stiffness constraint is a modeling constraint beyond which the aerodynamic loads for the analytical simulation are no longer valid, the probabilistic optimization cannot produce a feasible design with a $P_{r e q}$ that is greater than $1.07 \mathrm{e}-3$.

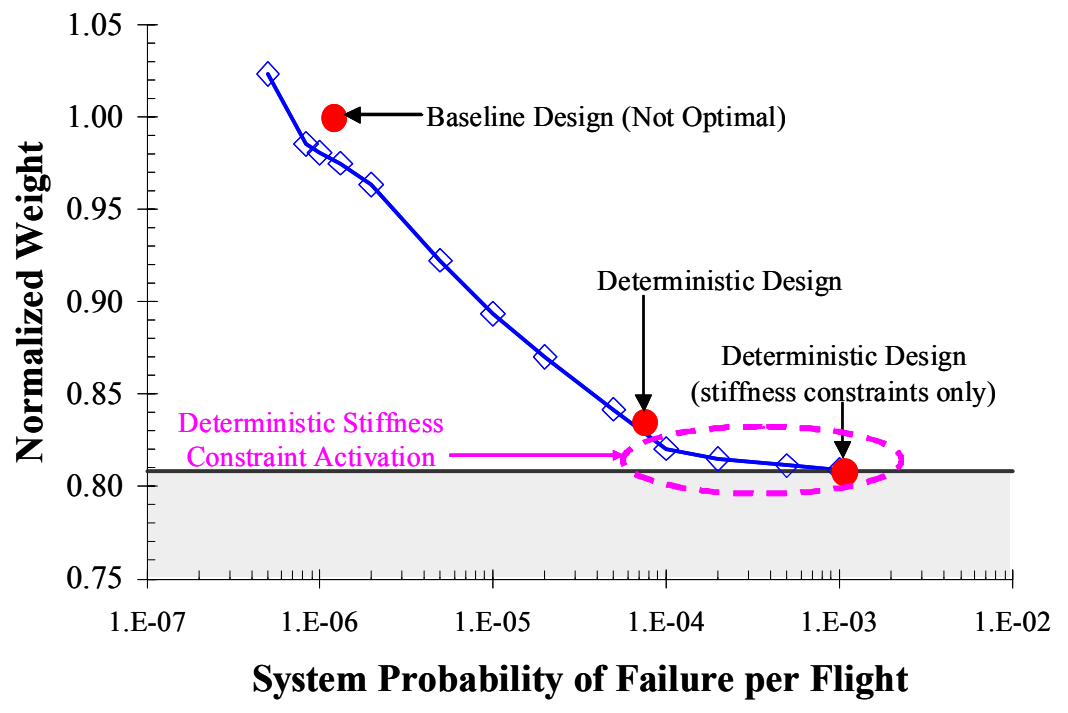

Figure 13. Optimum weights for several target probabilities of failure.

To put these results in perspective, consider the guidance that is provided by the Federal Aviation Administration (FAA) to certification engineers in the aircraft engine industry (Ref. 14). In FAR 25.1309 and other related advisory materials, the FAA defines a $P_{f}$ value in the range between $1.0 \mathrm{e}-5$ and $1.0 \mathrm{e}-7$ per hour as a "remote" probability of failure condition. A remote condition is defined as one that is unlikely to occur for any single aircraft during its lifetime but is likely to occur at least once in the lifetime of a fleet of aircraft. Ref. 14 explains that an acceptable $P_{f}$ value depends upon the severity of the effects that result from the failure condition. Because the RWT is secondary structure and the $P_{f}$ in this study describes relatively minor damage (first ply failure), the severity of condition effects for the RWT is in the "minor" category. Figure 14 (adapted from Refs. 14 and 15) implies that the baseline value of $P_{f}=1.0 \mathrm{e}-6$ per flight for the RWT is well within the acceptable range. 


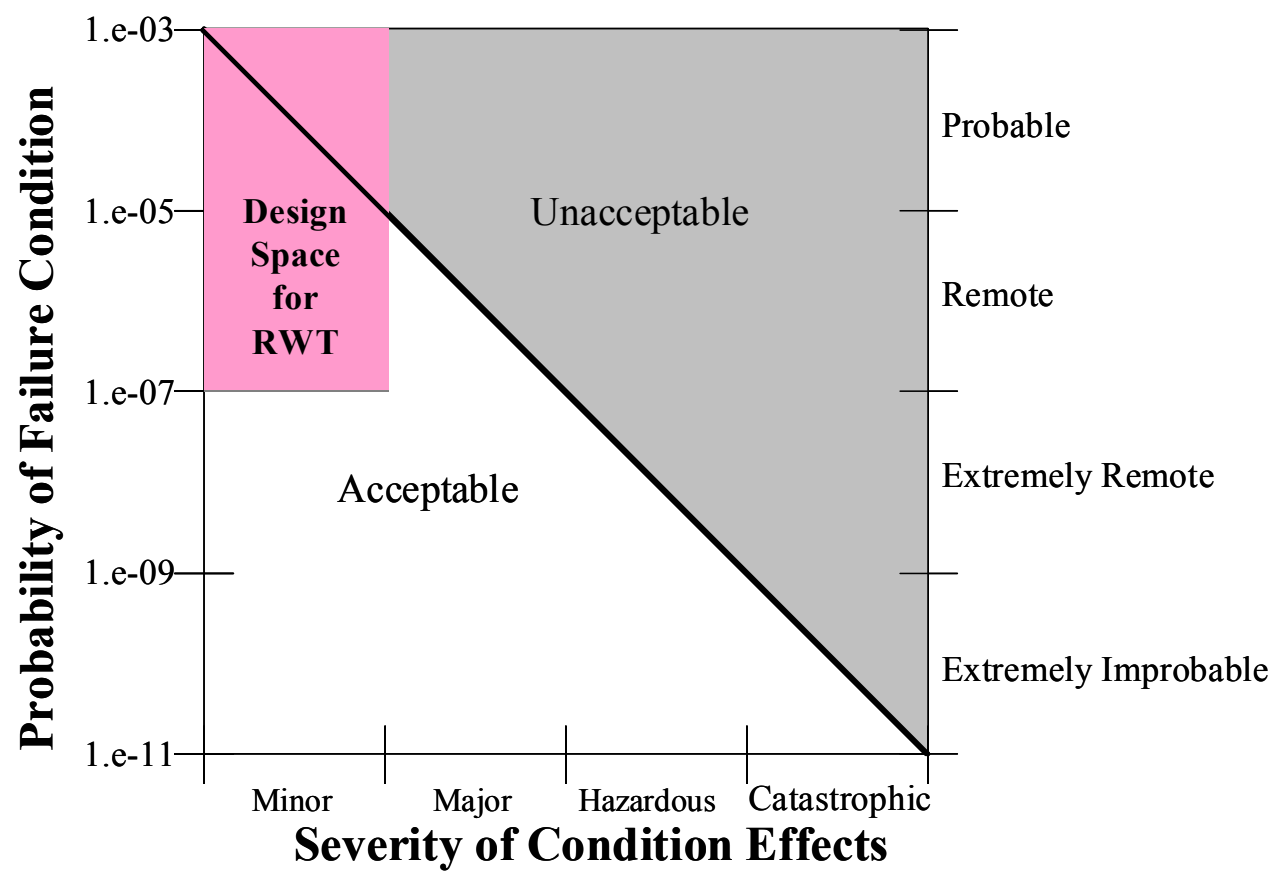

Figure 14. FAA Advisory guidance (where $1 \mathrm{e}-5<P_{f}<1 \mathrm{e}-7$ is considered to be Remote).

\section{Sensitivity Analysis}

A sensitivity analysis about the discrete optimum was performed, and a selected set of results is presented here. Only five of the thirteen design variables have values that are greater than the minimum gage for the discrete optimum. The sensitivities of the responses with respect to these five variables are shown in Figures 15 and 16. In Figure 15, the normalized weight is the weight divided by the baseline weight. In Figure 15, a one ply change in variable UC4 (upper cover thickness near the RWT root) results in a 0.73 percent change in the weight, which is larger than for any other variable. In Figure 16, the minimum MS among all of the elements in the upper cover for load case number 6 is evaluated against changes in the number of plies from the discrete optimum design. The MS response is nonlinear because the element in the FE model that has the minimum MS changes as the thickness changes. In Figure 16, a one ply change in variable UC4 results in a change in the MS of 0.036 in the vicinity of the optimum design (zero change in number of plies), which is significantly larger than for any other variable.

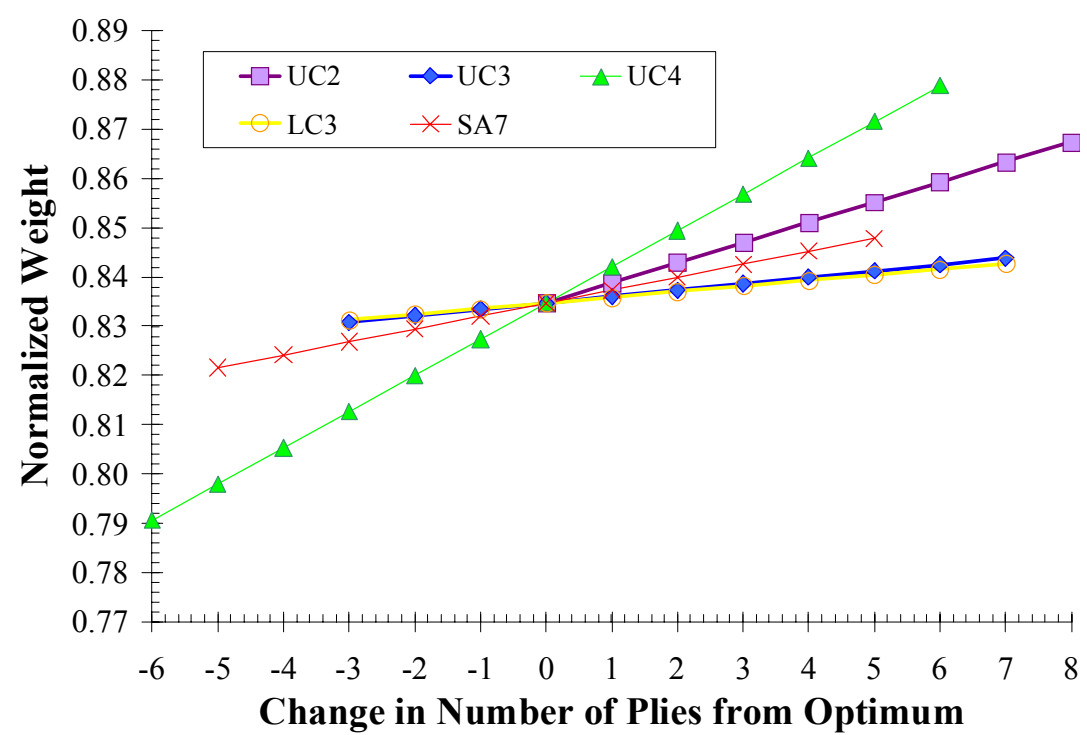

Figure 15. Weight sensitivity around the discrete deterministic optimum design. 


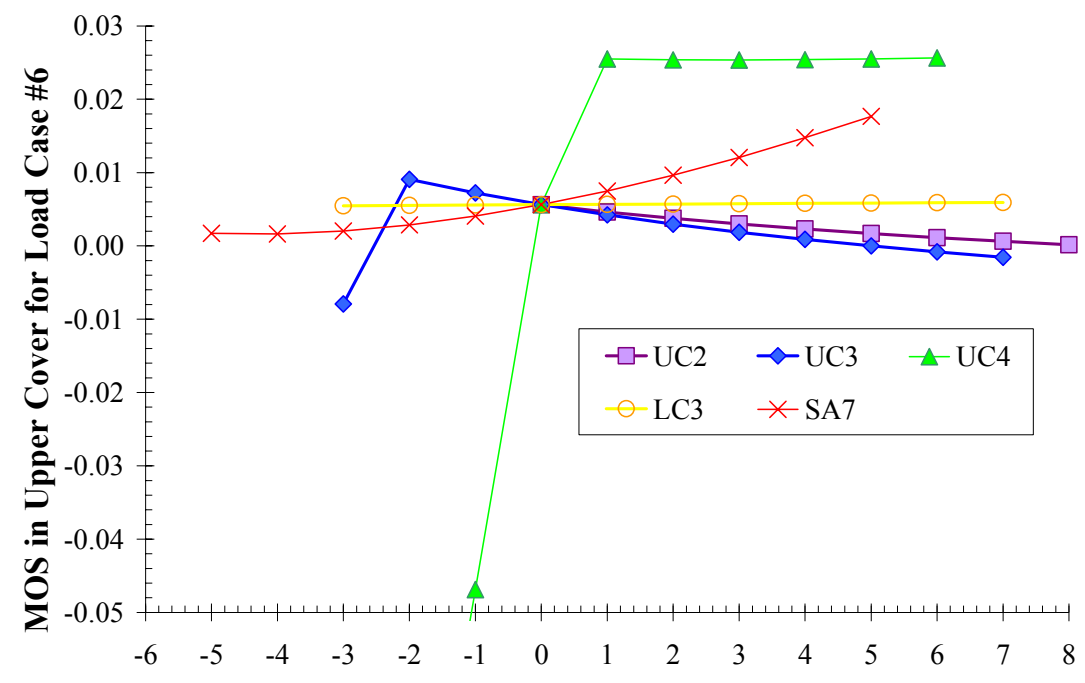

Change in Number of Plies from Optimum

Figure 16. MS sensitivity around the discrete deterministic optimum design.

In Figure 17, a two-dimensional cross section from the five-dimensional sensitivity study is shown. For these cross-sectional depictions of the design space, the two most important variables are selected: UC2 and UC4. Notice that for the discrete optimum, the torsional stiffness constraint is active, and the MS constraint is nearly active (very close to zero). Figure 17 demonstrates that if continuous design variables are considered, then the minimum weight optimum would occur at the intersection of the stiffness ratio and the MS constraints. Moreover, the MS constraint curve is nearly a horizontal line, which indicates that the design is primarily driven by the single variable UC4.

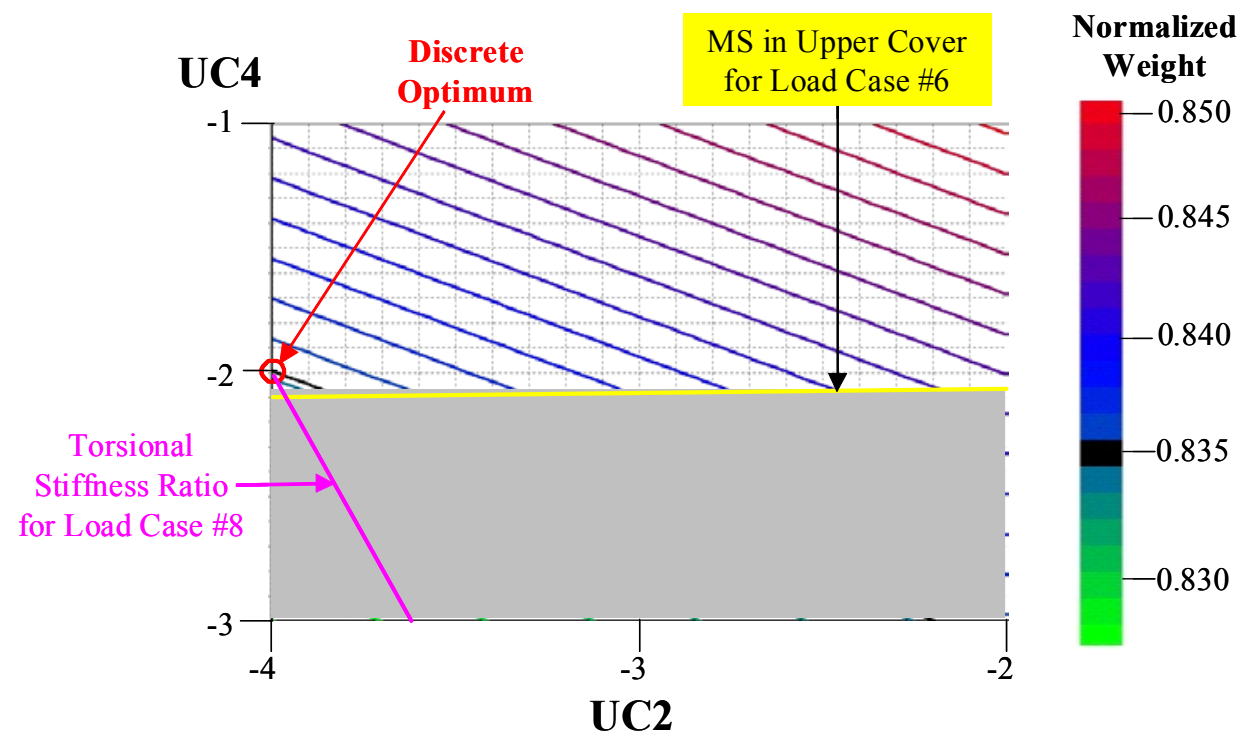

Figure 17. Weight contours for UC2-UC4 cross section of design space.

\section{Summary}

An approach for conducting reliability-based design and optimization (RBDO) of a Boeing 767 raked wing tip (RWT) is presented. Performance-based responses for the RWT are considered for eight critical aerodynamic load cases. Stiffness-based model constraints are used to steer the optimizer away from regions where the FE deformations may significantly affect the aerodynamic loading. For deterministic design, the performance constraint is the margin of safety (MS) that is computed by using the ultimate load. For probabilistic design, the 
performance constraint is the probability of failure $P_{f}$ that the MS is less than zero. In this section, the results of the RBDO study are discussed, and the lessons that have been learned from the RBDO process are presented.

\section{A. Discussion of Results for the RWT RBDO}

The first part of the RBDO process for the RWT was an evaluation of the baseline design. From a deterministic analysis, a MS of 0.126 was computed for the RWT under ultimate load. A deterministic optimization of the RWT, subject to the MS constraints, produced a design that weighed approximately 16 percent less than the baseline design. Of the 13 design variables that were considered, only five variables had values that were greater than the minimum gage for the deterministic optimum design. Of these five variables, the variable that controlled the thickness of the thickest plies in the upper cover was 20 times more influential than the next most critical variable.

For the probabilistic analysis and design, two sources of randomness were considered: randomness on the load scale factor and randomness in the strain limits. A normal distribution was established for the random load factor that predicts an occurrence of the limit load once per 3000 flights. Normal distributions were chosen for the strain limits based on typical and B-basis values and assuming a sample size between 5 and 30 . The MS for the metallic components were high, so the randomness was only considered for the strain limits in the composite laminates. For the baseline design with 8 random load factors and 28 random strain limits, the probability of failure was 1.2e-6. Several probabilistic optimizations were conducted with a target $P_{f}$ that ranged from 5.e-7 to 1.e-3. The optimized weight was a linear function of the $\log$ of $P_{f}$ between 5.e-7 and 1.e-4. For target values of $P_{f}$ above 1.e-4, the deterministic stiffness-based modeling constraint became active. This modeling constraint limited the feasible design for the probabilistic optimization to a maximum $P_{f}$ of $1.07 \mathrm{e}-3$ at a weight that is $80.44 \%$ of the baseline design.

\section{B. Lessons Learned from the probabilistic design process}

This sub section discusses the lessons learned during the RWT design study. The computational expense of RBDO is discussed first. Next, additional constraints that were not considered in this study are discussed. This section concludes with a discussion of the sources of uncertainty that were examined.

The most time-consuming part of this RBDO study was the time that is required to set up the optimization. Parameterization of the FE model required several weeks of work, particularly because of the hundreds of composite property cards, with up to a few dozen plies per card. Additionally, several passes were needed to develop a reasonable grouping of variables. Such tedious attention to detail is typical of the "art" of FE analysis. The outcome was a validated model that was used to explore the design space manually and with automated optimization and parameter studies.

Once the parameterization had been completed, setting up the deterministic optimization was quickly accomplished by using commercial framework software (e.g., ModelCenter), although a scripting language, such as PERL or UNIX shell scripts could also have been used. The software to compute a probabilistic constraint (using MC simulation or FORM) was a straightforward addition of a single "black box" to a framework. Some additional analysis time was required to perform a probabilistic calculation. For the current study, the deterministic analysis required less than 1 minute, and the probabilistic analysis required from a few minutes to a few hours, depending on the number of simulations that were required (for $\mathrm{MC}$ ) or the number of probabilistic constraints that were considered (for FORM). Most of the time that was required for the RBDO study was spent in activities that are needed regardless of whether the designer uses a traditional approach or a probabilistic and optimization-based approach.

A number of structural design criteria are used during the Boeing design process, but for this study, only stiffness and strength criteria were addressed. The deterministic stiffness constraint was used to keep the stiffness of the designed RWT within 10 percent of the baseline design. By applying this stiffness constraint, the aerodynamic loads were assumed to be constant, and the flutter behavior for the designed RWT was assumed to be the same as that of the baseline case. The NASTRAN model that was used in this study is only validated for static strength load cases; so only a first-ply failure criterion was used as a performance constraint. Several design criteria, were neglected because they were either not critical for the RWT component (e.g., crashworthiness and impact events) or because the design changes in this study would not affect these criteria (e.g., maintainability, producibility, and corrosion). Other criteria, such as fatigue and damage tolerance, are important for the RWT design, but it was necessary to exclude them from this study because the resources (such high fidelity FE models with threedimensional elements) and data (such as a dynamic load spectrum) were not available. However, the RBDO process could easily accommodate these criteria if these analytical models and data were available.

Accurate probability distributions for the primary sources of uncertainty in the RWT are important for probabilistic design. For the RWT design, two primary sources of uncertainty were noted: material strain limits and 
aerodynamic loads. In this study, the probabilistic distributions for the strain limits in the composite components of the RWT were developed from typical and B-basis values. Adjustments to the standard deviations for these material limits were made based on the number of coupon tests that were used to determine the B-basis values. The other primary source of uncertainty considered in this study was the randomness in the maximum loads experienced by the RWT. Because the loads were static, a probabilistic distribution was determined for a load scale factor in the FE model. This load scale factor represented uncertainties in the flight conditions (e.g., mission profile or weather) but did not represent uncertainties in the modeling or in the physics-based analysis codes. In this study, a stiffnessbased model constraint was used because the uncertainty in the modeling was difficult to quantify. Additional sources of uncertainty in material elastic properties and geometry were not considered in this study.

\section{Concluding Remarks}

One aim of the RWT design study was to compare the baseline design with the results of deterministic and probabilistic optimization. This study produced a chart that showed a clear relationship between the weight of the RWT and the system probability of failure per flight. The RWT study suggests that such a chart could be produced during the structural design process with data and knowledge that is already available to design engineers. A chart such as this is valuable because it provides an assessment of the baseline design and it predicts the reduction in reliability that would result from any mandated reduction in weight.

A second aim of the RWT study was to estimate the effort required to produce a probabilistic optimum design. The conclusion is that the added effort is small compared to the effort already required to produce and validate a parametric finite element model for trade studies. Use of probabilistic methods are especially recommended for components for which maintenance and operational data is available for characterizing the uncertainties in the loads and the material system. The probabilistic method provides important information concerning the design changes that will have the greatest effect on reliability for the minimum change in the design.

\section{References}

${ }^{1}$ Vanderplaats, Garret N.: "Industrial Applications of Optimization," 10th AIAA/ISSMO Multidisciplinary Analysis and Optimization Conference, Albany, NY, AIAA-2004-4505, August 30-September 1, 2004.

${ }^{2}$ Stroud, W. Jefferson, Krishnamurthy, T., Mason, Brian H., Smith, Steven A., and Naser, Ahmad S.: "Probabilistic Design of a Plate-Like Wing to Meet Flutter and Strength Requirements," 43rd AIAA/ASME/ASCE/AHS/ASC Structures, Structural Dynamics, and Materials Conference, Denver, CO, AIAA2002-1464, April 22-25, 2002.

${ }^{3}$ Mason, Brian H., Stroud, W. Jefferson, Krishnamurthy, T., Spain, Charles V., and Naser, Ahmad S.: "Probabilistic Design of a Wind Tunnel Model to Match the Response of a Full-Scale Aircraft," 46th AIAA/ASME/ASCE/AHS/ASC Structures, Structural Dynamics, and Materials Conference, Austin, TX, AIAA2005-2185, April 22-25, 2005.

${ }^{4}$ Haftka, Raphael T.: "Automated Procedure for Design of Wing Structures to Satisfy Strength and Flutter Requirements," NASA TN D-7264, 1973.

${ }^{5}$ Zang, Thomas A, Hemsch, Michael J., Hilburger, Mark W., Kenny, Sean P., Luckring, James M., Maghami, Peiman, Padula, Sharon L., and Stroud, W. Jefferson: "Needs and Opportunities for Uncertainty-Based Multidisciplinary Design Methods for Aerospace Vehicles," NASA/TM-2002-211462, July 2002.

${ }^{6}$ Caffrey, John P., and Lee, John N.: MSC/NASTRAN Linear Static Analysis, User's Guide, Version 68, The MacNeal-Schwendler Corporation, Los Angeles, CA, 1996.

${ }^{7}$ Tipps, Daniel O., Rustenburg, John W., and Skinn, Donald A.: "Statistical Loads Data for B-767-200ER Aircraft in Commercial Operations," DOT/FAA/AR-00/10, March 2000.

${ }^{8}$ Ang, Alfredo H.-S., and Tang, Wilson H.: Probability Concepts in Engineering Planning and Design. Vol. I, Basic Principles, John Wiley, 1975, pp. 98-101, 219-260.

${ }^{9}$ Ang, Alfredo H.-S., and Tang, Wilson H.: Probability Concepts in Engineering Planning and Design. Vol. II, Decision, Risk, and Reliability, John Wiley, 1984, pp. 206-216.

10“"Military Handbook - MIL-HDBK-17-1F: Composite Materials Handbook, Volume 1 - Polymer Matrix Composites Guidelines for Characterization of Structural Materials," U.S. Department of Defense, December 12, 2002.

${ }^{11}$ Anonymous, DOT, Design Optimization Tools, User's Manual, Version 5.0, Vanderplaats Research \& Development, Inc., Colorado Springs, CO 80906.

12،Process Integration Using ModelCenter ${ }^{\circledR}$," Technical White Paper, Phoenix Integration, Inc., Blacksburg, VA, 2000. 
${ }^{13}$ Park, Stephen K. and Miller, Keith W.: "Random Number Generators: Good Ones are Hard to Find," Communications of the ACM, Vol 31, No. 10, October 1988, pp. 1192-1201.

${ }^{14}$ Anonymous, "FAA Advisory Circular - AC-25.1309-1A: System Design and Analysis," U.S. Department of Transportation, June 21, 1988.

${ }^{15}$ Anonymous, "FAA Advisory Circular - AC-35.75-1A: Safety Analysis," U.S. Department of Transportation, September 26, 2007. 